\title{
Adsorption energy and spin state of first-row transition metals adsorbed on $\mathrm{MgO}(100)$
}

\author{
A. Markovits, ${ }^{1}$ J. C. Paniagua, ${ }^{2}$ N. López, ${ }^{2}$ C. Minot, ${ }^{1}$ and F. Illas ${ }^{2, *}$ \\ ${ }^{1}$ Laboratoire de Chimie Théorique, UMR 7616 UPMC/CNRS, boîte 137, Université Pierre et Marie Curie, 4 Place Jussieu, \\ F-75252 Paris cédex 05, France \\ ${ }^{2}$ Department de Química Física i Centre de Recerca en Química Teòrica, Universitat de Barcelona i Parc Científic de Barcelon, \\ C/Martí i Franquès 1, E-08028 Barcelona, Spain
}

(Received 18 October 2002; published 31 March 2003)

\begin{abstract}
Slab and cluster model spin-polarized calculations have been carried out to study various properties of isolated first-row transition metal atoms adsorbed on the anionic sites of the regular $\mathrm{MgO}(100)$ surface. The calculated adsorption energies follow the trend of the metal cohesive energies, indicating that the changes in the metal-support and metal-metal interactions along the series are dominated by atomic properties. In all cases, except for $\mathrm{Ni}$ at the generalized gradient approximation level, the number of unpaired electron is maintained as in the isolated metal atom. The energy required to change the atomic state from high to low spin has been computed using the PW91 and B3LYP density-functional-theory-based methods. PW91 fails to predict the proper ground state of $\mathrm{V}$ and $\mathrm{Ni}$, but the results for the isolated and adsorbed atom are consistent within the method. B3LYP properly predicts the ground state of all first-row transition atom the high- to low-spin transition considered is comparable to experiment. In all cases, the interaction with the surface results in a reduced high- to low-spin transition energy.
\end{abstract}

DOI: 10.1103/PhysRevB.67.115417

PACS number(s): 73.20.At, 71.15.Mb, 68.47.Gh

\section{INTRODUCTION}

The study of the interaction between metal and metal oxide surfaces has been extensively carried out from experimental ${ }^{1}$ and theoretical points of view ${ }^{2}$ because of its prime importance in many industrial applications ranging from microelectronic devices to heterogeneous catalysis. ${ }^{3-6}$ Among the different supports, the $\mathrm{MgO}(100)$ surface has long been used as a model support for surface science studies because of its stability, low reactivity and simple structure. Nevertheless, the interaction of metals on the non-defective $\mathrm{MgO}(100)$ surface is weaker than ordinary chemical bonds and difficult to describe, especially for transition-metal atoms. The difficulty in characterizing the strength of the metal-support interaction is found from both experimental and theoretical sides. On the former, the complexity comes from the insulating character of the oxides surfaces but also from the difficulty to control the quality of the oxide surface and the structure of the adsorbate. ${ }^{7,8}$ From the theoretical side the situation is not better because the adsorption energy turns out to be strongly dependent on the method chosen to compute the total energy. Thus it is now well established that the local-density approximation to the exchange-correlation functional of density functional theory largely overestimates the adsorption energy, whereas the generalized gradient approximation and gradient-corrected techniques have a better, albeit nonperfect, behavior. ${ }^{9}$ On the other hand, methods based on explicitly correlated wave functions provide highly accurate results, but in this context their use is restricted to the cluster model representation of the surface and, in practice, can only be applied to models containing a rather small number of atoms. ${ }^{10,11}$

Although a systematic study for the first-row transitionmetal series is still lacking, previous studies classified the transition-metal atoms into two main groups. ${ }^{12,13}$ According to Yudanov et al. ${ }^{12}$ the first group involves atoms which tend to form moderately strong chemical bonds with the surface oxygen anions of $\mathrm{MgO}(\mathrm{Ni}, \mathrm{Pd}, \mathrm{Pt}$, and $\mathrm{W})$, and those with very weak interaction with the surface $(\mathrm{Cr}, \mathrm{Mo}, \mathrm{Cu}, \mathrm{Ag}$, and $\mathrm{Au}$ ). In the former group adsorption energies are of the order of $1 \mathrm{eV}$, whereas in the later adsorption energies are at least three times smaller. The different strength of the interaction is due to the metal-substrate covalent bonding that also implies a polarization of the metal orbitals (a redistribution of the atomic orbital population). In all cases, the metal $s$ orbital combines with the oxygen $p$ orbital perpendicular to the surface resulting in a bonding (occupied) and an antibonding (empty) combination; this leads to a decrease in the atomic $s$ population for the metal adatom. When the free atom electronic configuration is $d^{n} s^{2}$, the resulting electronic configuration of the adatom can be described as $\sim d^{n+1} s^{1}$, although rigorously speaking one should not use this notation because the atomic symmetry is lost. The strength of the metal-oxide interaction varies with the resulting $d$ population. This change in the electronic configuration of the adsorbed atom may result in a concomitant spin quenching with respect to ground state multiplicity of the isolated transition metal atom. Notice that the perfect $\mathrm{MgO}(100)$ surface has not any net spin. Hence, the resulting spin arises solely from the adsorbed transition metal atom. The low lying electronic states of $\mathrm{Ni}$ on $\mathrm{MgO}$ (100) have been studied in detail for $\mathrm{Ni}$ on basic sites of the perfect substrate ${ }^{14}$ and for various oxygen vacancies point defects on the same surface. ${ }^{15}$ In the later case, situations with different coverage were also discussed.

The study of Ni on perfect and defective $\mathrm{MgO}(100)$ surfaces has permitted one to note several important trends concerning the final magnetic properties of the adsorbed atom on thin film. As a rule of thumb, the stronger the interaction the larger the spin quenching and, consequently, the higher the low- to high-spin state energy transition. For strong interactions, the final electronic structure corresponds to a singlet 
state with no net magnetic moment on the adatom. This is in line with the results reported in the systematic work of Yudanov et al. ${ }^{12}$ However, while the relationship between adsorption energy and final adatom spin state is observed regardless of the exchange-correlation functional used in the DFT calculations, the adsorption energy appears to be strongly method dependent, ${ }^{10}$ but shows very little dependence on the choice of a cluster or a slab representation of the $\mathrm{MgO}(100)$ surface. ${ }^{11}$ Although Ni is probably a singular case in the first-row transition-metal atoms because of the quasidegeneracy of the $d^{8} s^{2}$ and $d^{9} s^{1}$ atomic electronic configurations, it seems necessary to further investigate the magnetic properties of first-row transition metal atoms on $\mathrm{MgO}(100)$. Likewise, it is important to complete the picture drawn by Yudanov et al. ${ }^{12}$ by extending the study to the first row of transition-metal atoms. These are precisely the goals of the present paper.

\section{COMPUTATIONAL DETAILS}

This paper tries to address two important points concerning the interaction of transition metal atoms with a simple oxide surface. On the one hand, the need for a spin-polarized treatment to properly describe the ground-state electronic structure and adsorption energies, and on the other hand to give an estimate of the low- to high-spin energy transition. For the former one can use either a cluster or a slab model with similar results. ${ }^{11}$ However, it is more appropriate to use a slab approach provided spin polarization is included, thus avoiding problems related to the embedding or to the basis set superposition error. For the latter both models can in principle be used. Nevertheless, in the slab calculation one can only fix the number of unpaired electrons (per unit cell) whereas in the cluster approach it is possible to better control the electronic configuration although in any case the use of an unrestricted formalism does not always allow a proper description of some open shell electronic states. In this paper, the above points have been carefully analyzed for the adsorption of the first row transition metals on the basic (anionic) sites of the $\mathrm{MgO}(100)$ surface. For the regular surface this is known to be the most favorable adsorption site. ${ }^{2,12,16}$

In the slab approach, the calculation is periodic in three dimensions. Two vectors reproduce the $\mathrm{MgO}(100)$ unit cell while the third one, perpendicular to the surface, is used to represent a slab of the material and to leave a vacuum width between two successive slabs. The slab is made of three atomic layers, and the vacuum width is chosen to be large enough so as to prevent any interaction between the slabs. In the cluster approach the model representing the oxygen $\mathrm{MgO}(100)$ adsorption site is stoichiometric and contains nine oxygen and nine magnesium atoms which are surrounded by total ion potentials (TIPs) to avoid unphysical polarization effects. The TIPs replace the Mg cations in direct contact to the edge oxygen atoms. The cluster plus TIPs is further embedded in a $16 \times 16 \times 4$ array of \pm 2 point charges which provide the adequate Madelung potential. For the regular surface the entire system, cluster plus PCs, is neutral. A more detailed discussion has been given elsewhere. ${ }^{14}$

All calculations have been carried out in the framework of
DFT. The periodic and cluster DFT calculations have been performed using the GGA method proposed by Perdew and Wang ${ }^{17,18}$ and hereafter referred to as PW91. Cluster calculations have been also repeated using the well-known hybrid B3LYP functional. ${ }^{19}$ The use of different functionals is necessary because of the strong dependence of the adsorption and of the low- to high-spin energy transition energies on the chosen functional. Notice that even for the free metal atom, the results depend on the method of calculation. For instance, the PW91 ground state for $\mathrm{Ni}$ is $d^{9} s^{1}$ instead of $d^{8} s^{2}$, which is the experimental ground state and that appears at too high an energy in the PW91 calculations. ${ }^{20} \mathrm{~A}$ wrong description of the free atom foresees a possible false prediction of the final magnetic moment of the adsorbed nickel atom.

For the periodic calculations, a plane wave basis set with a cutoff of $396 \mathrm{eV}$ has been used to describe the $4 s$ and $3 d$ electrons of the metals and the valence electrons of $\mathrm{MgO}$. For $\mathrm{Sc}, \mathrm{Ti}$, and $\mathrm{V}$ the $3 p$ electrons have also been treated in the valence shell. The core electrons are replaced by ultrasoft pseudopotentials. ${ }^{21,22}$ A Monkhorst-Pack grid consisting of $5 \times 5 \times 1$ special $k$ points has been employed to perform the integration in the reciprocal space. Contracted Gaussian type orbitals (CGTOs) have been employed in the cluster model calculations. For the five $\mathrm{Mg}$ atoms surrounding the oxygen adsorption site the basis set consists of a $6 s 3 p$ contraction of a $13 s 8 p$ primitive set; this is indicated as $[13 s 8 p / 6 s 3 p]$. For the remaining $\mathrm{Mg}$ atoms a smaller $[12 s 7 p / 5 s 2 p]$ basis set has been used. For the $\mathrm{O}$ atom directly interacting with adsorbate the basis set is $[8 s 4 p 1 d / 4 s 2 p 1 d]$ whereas that corresponding to the remaining oxygen atoms is $[8 s 4 p / 4 s 2 p]$. For the transition metal atoms we have employed the small core relativistic pseudopotentials proposed by Hay and Wadt. ${ }^{23}$ However, to allow a better description of their electronic structure, the primitive Hay and Wadt basis set has been left uncontracted. Further details about the CGTO basis sets can be found in previous work. ${ }^{10,11,14}$ The periodic and cluster calculations have been carried out using the Vienna $A b$ initio Simulation Program (VASP), ${ }^{24-26}$ and the GAUSSIAN98 (Ref. 27) suite of programs, respectively.

In the periodic calculations a geometry optimization has been carried out for the perpendicular distance of the metal atom above the surface, and all substrate atoms have been frozen to their experimental position in the bulk. In these calculations, the unit cell contains eight surface oxygen per adsorbed transition-metal atom, resulting in a coverage of $\theta$ $=\frac{1}{8}$. In this way, the adsorbate-adsorbate distance is $8.42 \AA$, which is large enough so as to prevent any lateral interaction. Indeed, this low coverage facilitates the comparison with the cluster results. The adsorption energies have been calculated as

$$
E_{\mathrm{ads}}=E(\mathrm{TM})+E(\mathrm{MgO})-E(\mathrm{TM} / \mathrm{MgO})
$$

where $E(\mathrm{TM})$ is the spin-polarized energy of the transition metal atom for the electronic configuration given in Table I and is obtained by placing the atom in a cubic box of $10 \AA^{3}$, $E(\mathrm{MgO})$ is the energy of the bare $\mathrm{MgO}$ slab and $E(\mathrm{TM} / \mathrm{MgO})$ is the energy of the supersystem in the elec- 
TABLE I. Experimental and calculated (PW91 and B3LYP within the GTO basis set) ground state of the first row transition metal atoms. Since in the spin unrestricted calculations the atomic symmetry is not preserved only the apparent electronic configuration and the number of unpaired electrons are given. Notice that experimental and PW91 ground states for V and Ni are different.

\begin{tabular}{|c|c|c|c|c|c|}
\hline & \multirow[b]{2}{*}{$\begin{array}{l}\text { Experimental } \\
\text { ground state }\end{array}$} & \multicolumn{2}{|c|}{$\begin{array}{l}\text { Calculated PW91 } \\
\text { ground state }\end{array}$} & \multicolumn{2}{|c|}{$\begin{array}{l}\text { Calculated B3LYP } \\
\text { ground state }\end{array}$} \\
\hline & & $\begin{array}{l}\text { Electronic } \\
\text { conf. }\end{array}$ & $\begin{array}{l}\text { Unpaired } \\
\text { electrons }\end{array}$ & $\begin{array}{l}\text { Electronic } \\
\text { conf. }\end{array}$ & $\begin{array}{l}\text { Unpaired } \\
\text { electrons }\end{array}$ \\
\hline $\mathrm{Sc}$ & $\ldots 3 d^{1} 4 s^{2}\left({ }^{2} D\right)$ & $\ldots 3 d^{1} 4 s^{2}$ & 1 & $\ldots 3 d^{1} 4 s^{2}$ & 1 \\
\hline $\mathrm{Ti}$ & $\ldots 3 d^{2} 4 s^{2}\left({ }^{3} F\right)$ & $\ldots 3 d^{2} 4 s^{2}$ & 2 & $\ldots 3 d^{2} 4 s^{2}$ & 2 \\
\hline $\mathrm{V}$ & $\ldots 3 d^{3} 4 s^{2}\left({ }^{4} F\right)$ & $\ldots 3 d^{4} 4 s^{1}$ & 5 & $\ldots 3 d^{3} 4 s^{2}$ & 3 \\
\hline $\mathrm{Cr}$ & $\ldots 3 d^{5} 4 s^{1}\left({ }^{7} S\right)$ & $\ldots 3 d^{5} 4 s^{1}$ & 6 & $\ldots 3 d^{5} 4 s^{1}$ & 6 \\
\hline Mn & $\ldots 3 d^{5} 4 s^{2}\left({ }^{6} S\right)$ & $\ldots 3 d^{5} 4 s^{2}$ & 5 & $\ldots 3 d^{5} 4 s^{2}$ & 5 \\
\hline $\mathrm{Fe}$ & $\ldots 3 d^{6} 4 s^{2}\left({ }^{5} D\right)$ & $\ldots 3 d^{6} 4 s^{2}$ & 4 & $\ldots 3 d^{6} 4 s^{2}$ & 4 \\
\hline $\mathrm{Co}$ & $\ldots 3 d^{7} 4 s^{2}\left({ }^{4} F\right)$ & $\ldots 3 d^{7} 4 s^{2}$ & 3 & $\ldots 3 d^{7} 4 s^{2}$ & 3 \\
\hline $\mathrm{Ni}$ & $\ldots 3 d^{8} 4 s^{2}\left({ }^{3} F\right)$ & $\ldots 3 d^{9} 4 s^{1}$ & 2 & $\ldots 3 d^{8} 4 s^{2}$ & 2 \\
\hline $\mathrm{Cu}$ & $\ldots 3 d^{10} 4 s^{1}\left({ }^{2} S\right)$ & $\ldots 3 d^{10} 4 s^{1}$ & 1 & $\ldots 3 d^{10} 4 s^{1}$ & 1 \\
\hline $\mathrm{Zn}$ & $\ldots 3 d^{10} 4 s^{2}\left({ }^{1} S\right)$ & $\ldots 3 d^{10} 4 s^{2}$ & 0 & $\ldots 3 d^{10} 4 s^{2}$ & 0 \\
\hline
\end{tabular}

tronic ground state (see below). With this choice, positive adsorption energies correspond to exothermic processes.

In the cluster calculations the procedure described above for the periodic calculations has also been used but atomic calculations have been carried out for the lowest energy configuration with low- and high-spin couplings. The same procedure has been used in the case of adsorbed metal atoms. In order to investigate the effect of the computational method all cluster calculations have been carried out at the unrestricted PW91 and B3LYP levels of theory.

\section{RESULTS AND DISCUSSION}

\section{A. Adsorption energies}

The periodic PW91-slab spin-polarized adsorption energies and perpendicular distance to the surface for the firstrow transition-metal atoms above the basic sites of $\mathrm{MgO}(100)$ are summarized in Table II and Fig. 1. The results for $\mathrm{K}$ and $\mathrm{Ca}$ are also indicated for comparison purposes. All adsorption energies range from $0.1 \mathrm{eV}(\mathrm{Zn})$ to $1.3 \mathrm{eV}(\mathrm{Ni})$, which are in good agreement with previous cluster model DFT studies. ${ }^{12}$ Notice that, as expected, there is an inverse correlation between adsorption energy and equilibrium distance, the larger the former the shorter the later. The change of the values is far from being monotonous as expected from the complex electronic structure of these metal atoms.

On the other hand, the adsorption energies closely follow the trend of the cohesive energies of the bulk (see Fig. 1.1 and Ref. 15 in Ref. 28). PW91-slab calculations place the second maximum on $\mathrm{Ni}$ as the experimental curve, while the muffin-tin LDA calculations provides a shift to Co. Interestingly enough the same trend has been deduced from microcalorimetric measurements. ${ }^{29}$ The interaction of a monolayer of second-row transition metals on $\mathrm{MgO}$ does also follow this trend ${ }^{13}$ although in this case the total energy is obtained
TABLE II. Calculated spin-polarized PW91-slab perpendicular distance to the surface $\left(r_{e}\right)$, adsorption energies $\left(E_{\text {ads }}\right)$ and number of unpaired electrons per unit cell in the electronic ground state $\left(N_{\alpha}-N_{\beta}\right)$. Notice that for Co there are two nearly degenerate states.

\begin{tabular}{cccc}
\hline \hline Atom & $r_{e}(\AA)$ & $E_{\text {ads }}(\mathrm{eV})$ & $N_{\alpha}-N_{\beta}$ \\
\hline $\mathrm{K}$ & 3.292 & 0.145 & 1 \\
$\mathrm{Ca}$ & 2.586 & 0.333 & 0 \\
$\mathrm{Sc}$ & 2.215 & 0.809 & 1 \\
$\mathrm{Ti}$ & 2.050 & 1.213 & 2 \\
$\mathrm{~V}$ & 2.162 & 1.014 & 5 \\
$\mathrm{Cr}$ & 2.424 & 0.399 & 6 \\
$\mathrm{Mn}$ & 2.148 & 0.596 & 5 \\
$\mathrm{Fe}$ & 1.969 & 0.805 & 4 \\
$\mathrm{Co}$ & 1.890 & 0.802 & 1 \\
& 1.987 & 0.798 & 3 \\
$\mathrm{Ni}$ & 1.823 & 1.327 & 0 \\
$\mathrm{Cu}$ & 2.032 & 0.935 & 1 \\
$\mathrm{Zn}$ & 3.094 & 0.094 & 0 \\
\hline \hline
\end{tabular}

from a non-spin-polarized method, and consequently the adsorption energy does not refer to the atom. In forthcoming discussion we will suggest a possible explanation to this observation. However, it is important to realize that there is an essential difference between the present calculations and those reported in Ref. 13. The present study has been carried out at the spin-polarized level, a choice which permits a better albeit non perfect estimate of the atomic reference (see below). In any case, the parallelism between adsorption and cohesive energies indicates that the underlying physics dominating the changes in metal-oxygen and metal-metal interactions along the series is the same.

\section{B. Low-lying spin states of adsorbed atoms}

For all first-row transition metals the slab and cluster calculations indicate that, at the GGA level, the number of unpaired electrons in the adatoms does not change under adsorption with respect to that of the isolated atom (see Tables I, II, and III). Notice, however, that for the atom in gas phase the PW91 description for $\mathrm{V}$ and $\mathrm{Ni}$ is wrong. In both cases the ground state electronic configuration is different from experiment and in the case of $\mathrm{V}$ the multiplicity is also wrong (see Table I).

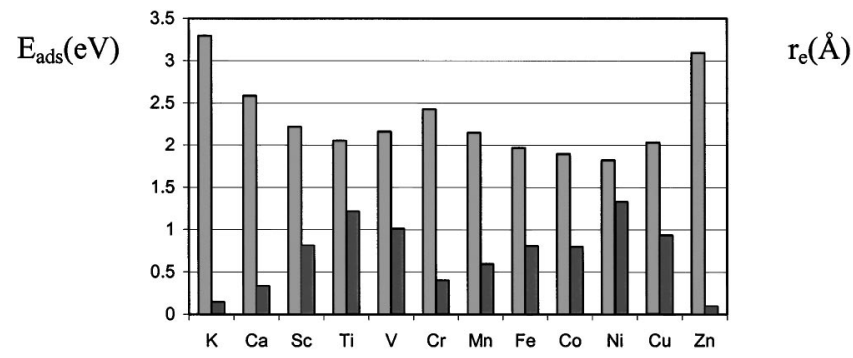

FIG. 1. Calculated PW91-slab adsorption energies (dark bars, right) and the metal-oxygen distances (light bars, left). 
TABLE III. Number of unpaired electrons $\left(N_{\alpha}-N_{\beta}\right)$ in the high- (HS) and low-spin (LS) states of the adsorbed transition-metal atoms. Transition energy $\left(\Delta E_{\text {adsorbed }}^{H-L}\right)$ required to excite adsorbed transition metal atom from the high- to low-spin as obtained in the cluster model calculations. A negative sign indicates that the ground state is provided by the low-spin coupling. The last column of the table reports the change in the equilibrium distance perpendicular to the surface $\left(\Delta r_{e}^{H-L}\right)$ when going from high- to low-spin state. A positive value indicates that $r_{e}$ is larger in the high-spin state.

\begin{tabular}{|c|c|c|c|c|c|}
\hline Atom & $\begin{array}{l}N_{\alpha}-N_{\beta} \\
\quad(\mathrm{HS})\end{array}$ & $\begin{array}{l}N_{\alpha}-N_{\beta} \\
\quad(\mathrm{LS})\end{array}$ & Method & $\begin{array}{c}\Delta E_{\text {adsorbed }}^{H-L} \\
\quad(\mathrm{eV})\end{array}$ & $\begin{array}{c}\Delta r_{e}^{H-L} \\
(\AA)\end{array}$ \\
\hline \multirow[t]{2}{*}{$\mathrm{Ti}$} & \multirow[t]{2}{*}{2} & \multirow[t]{2}{*}{0} & PW91 & 0.651 & 0.175 \\
\hline & & & B3LYP & 0.842 & 0.217 \\
\hline \multirow[t]{2}{*}{$\mathrm{V}$} & \multirow[t]{2}{*}{5} & \multirow[t]{2}{*}{3} & PW91 & 0.270 & 0.076 \\
\hline & & & B3LYP & -0.066 & 0.073 \\
\hline \multirow[t]{2}{*}{$\mathrm{Cr}$} & \multirow[t]{2}{*}{6} & \multirow[t]{2}{*}{4} & PW91 & 0.297 & 0.279 \\
\hline & & & B3LYP & 0.141 & 0.292 \\
\hline \multirow[t]{2}{*}{$\mathrm{Mn}$} & \multirow[t]{2}{*}{5} & \multirow[t]{2}{*}{3} & PW91 & 1.002 & 0.248 \\
\hline & & & B3LYP & 1.363 & 0.298 \\
\hline \multirow[t]{2}{*}{$\mathrm{Fe}$} & \multirow[t]{2}{*}{4} & \multirow[t]{2}{*}{2} & PW91 & 1.109 & 0.059 \\
\hline & & & B3LYP & 1.185 & 0.010 \\
\hline \multirow[t]{2}{*}{$\mathrm{Co}$} & \multirow[t]{2}{*}{3} & \multirow[t]{2}{*}{1} & PW91 & 0.447 & 0.044 \\
\hline & & & B3LYP & -0.056 & -0.042 \\
\hline \multirow[t]{2}{*}{$\mathrm{Ni}$} & \multirow[t]{2}{*}{2} & \multirow[t]{2}{*}{0} & PW91 & 0.300 & 0.220 \\
\hline & & & B3LYP & -0.020 & 0.281 \\
\hline
\end{tabular}

With respect to the cluster-slab comparison, several points merit a more detailed discussion. First, the adsorption energies $\left(E_{\text {ads }}\right)$ obtained with the cluster model and corrected by the basis set superposition error are very close to those predicted from the periodic calculations. The largest difference is $\sim 0.5 \mathrm{eV}$, which is not negligible, it is about $50 \%$ of $E_{\text {ads }}$. However, a large part of the energy difference arises from the difference in the description of the isolated atom. This point has been described in detail for Ni (Ref. 14) and will not be further discussed here. Second, the perpendicular distance to the surface predicted by both models is within $0.04 \AA$ except for Sc and Cr, where the difference is larger. Nevertheless, the main point of this paper is not a detailed comparison of the slab and cluster representation of the $\mathrm{MgO}(100)$ surface but rather the energy difference between high- and low-spin states of adsorbed transition-metal atoms.

The next point of the present study concerns the dependence of the number of unpaired electrons in the adsorbed atom with respect to the functional chosen. At this point it is important to realize that the accurate computation of atomic energy transitions is quite demanding. This is a rather intricate property requiring the use large configuration interaction wave functions. Notice that second-order perturbation theory calculations based on a complete active space selfconsistent-field wave function using large atomic basis sets predict excitation energies that are in error by as much as $\sim 0.2 \mathrm{eV}^{30}$ Density functional theory based methods are in general less accurate. ${ }^{31}$ This is clearly seen from results in Table IV, which reports the experimental high- to low-spin energy transition $\left(\Delta E_{\text {atomic }}^{H-L}\right)$ for isolated atoms where magnetic properties are relevant ( $\mathrm{Ti}$ to $\mathrm{Ni}$ ) and the values pre-
TABLE IV. Number of unpaired electrons $\left(N_{\alpha}-N_{\beta}\right)$ in the high- (HS) and low-spin (LS) states for the isolated transition metal atoms. Transition energy $\left(\Delta E_{\text {atomic }}^{H-L}\right)$ required to excite from the high to low spin. A negative sign indicates that the ground state is provided by the low-spin coupling. Calculated values have been obtained using the GTO basis.

\begin{tabular}{|c|c|c|c|c|c|}
\hline Atom & $\begin{array}{c}N_{\alpha}-N_{\beta} \\
\quad(\mathrm{HS})\end{array}$ & $\begin{array}{l}N_{\alpha}-N_{\beta} \\
\quad(\mathrm{LS})\end{array}$ & $\begin{array}{c}\Delta E_{\text {atomic }}^{H-L} \\
(\mathrm{eV}) \\
\text { Expt. values }\end{array}$ & $\begin{array}{c}\Delta E_{\text {atomic }}^{H-L} \\
(\mathrm{eV}) \\
\text { PW91 }\end{array}$ & $\begin{array}{c}\Delta E_{\text {atomic }}^{H-L} \\
(\mathrm{eV}) \\
\text { B3LYP }\end{array}$ \\
\hline $\mathrm{Ti}$ & 2 & 0 & 0.874 & 1.706 & 1.542 \\
\hline V & 5 & 3 & -0.245 & 0.485 & -0.060 \\
\hline $\mathrm{Cr}$ & 6 & 4 & 0.941 & 1.218 & 0.793 \\
\hline $\mathrm{Mn}$ & 5 & 3 & 2.915 & 1.814 & 3.234 \\
\hline $\mathrm{Fe}$ & 4 & 2 & 1.488 & 1.432 & 1.673 \\
\hline $\mathrm{Co}$ & 3 & 1 & 0.879 & 0.559 & 0.837 \\
\hline $\mathrm{Ni}$ & 2 & 0 & 1.826 & 4.730 & 3.000 \\
\hline
\end{tabular}

dicted from the PW91 and B3LYP functionals. Sc and $\mathrm{Cu}$ are not included because there is no possible spin quenching governed by a change in the atomic electronic configuration. The same occurs for $\mathrm{Zn}$ which indeed has no net magnetic moment, neither in the atomic ground state nor on the adsorbed state. The experimental values have been taken from the web database at the National Institute of Standards and Technology. ${ }^{32}$ The number of unpaired electrons in each state is indicated in Table IV, and the corresponding ground state electronic configuration is that reported in Table I. The results in Table IV show that the PW91 functional provides a qualitatively, and almost semiquantitative, correct description of $\Delta E_{\text {atomic }}^{H-L}$ except for $\mathrm{V}$ and Ni. In both cases the error comes from the incorrect prediction of the ground state electronic configuration (cf. Table I). In the case of V there is a change in the configuration and in the multiplicity and in the case of Ni only in the configuration because both $d^{8} s^{2}$ and $d^{9} s^{1}$ lowest multiplet is a triplet; see discussion in Ref. 14 . The configuration change in $\mathrm{V}$ at the GGA could be anticipated from the previous study of Baerends et al. ${ }^{31}$ However, the B3LYP result for $\mathrm{V}$ and $\mathrm{Ni}$ is also qualitatively correct and the excitation energies closer to experiment, as clearly shown in Fig. 2. Moreover, the B3LYP description for the rest of atoms is very similar to that commented above for the PW91 functional except that on the average the transition energies are closer to experiment. This is clearly illustrated in Fig. 2, which plots the calculated values in front of the experimental ones, the black line corresponding to an ideal perfect agreement between theory and experiment. In the context of atomic excitation energies the performance of B3LYP is remarkable.

Table III reports results for the high- to low-spin transition of the transition metal atoms but once adsorbed on $\mathrm{MgO}(100), \Delta E_{\text {adsorbed }}^{H-L}$. For $\mathrm{V}$ two different transitions have been considered because its ground state (either experimental or B3LYP) is a quartet state. Consequently, the transition from quartet to doublet has also been computed. The comparison between Tables III and IV permits one to conclude that, upon interaction with the $\mathrm{MgO}(100)$ surface, the number of unpaired electrons is preserved except perhaps for $\mathrm{V}$, 


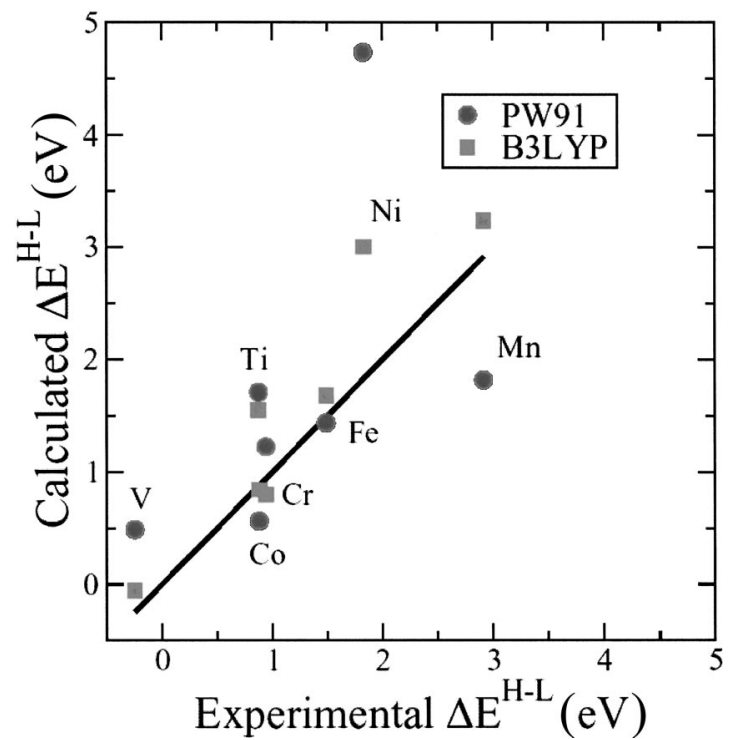

FIG. 2. Calculated vs experimental values for the high- to lowspin state transition energy $\left(\Delta E^{H-L}\right)$ for the free atoms. Notice that the solid line is the reference for a complete agreement between theory and experiment.

$\mathrm{Co}$, and $\mathrm{Ni}$, where according to the more reliable B3LYP method the low-spin state is slightly favored. In the case of $\mathrm{V}$ the transition to the doublet state requires $0.80 \mathrm{eV}$, this is somehow lower than the corresponding value for the isolated atom $(0.94 \mathrm{eV})$ but in any case the quartet state is the ground state for both isolated and adsorbed cases. It is interesting to compare $\Delta E_{\text {atomic }}^{H-L}$ to $\Delta E_{\text {adsorbed }}^{H-L}$ which provides information of the change in the transition energy induced by the surface. This comparison is reported in Fig. 3, which shows that in all cases the energy required to go from the high-spin to the

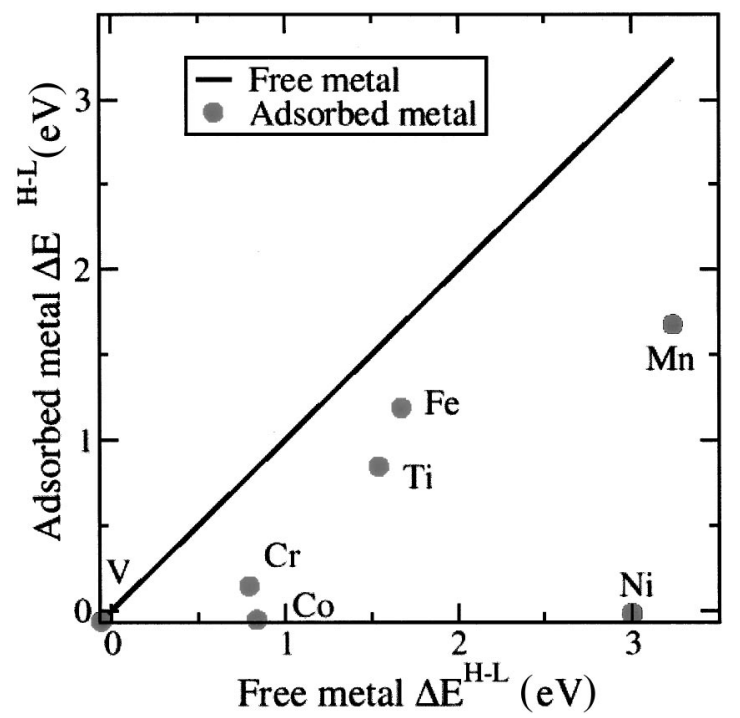

FIG. 3. B3LYP values for the high- to low-spin state transition energy for the adsorbed atom versus the corresponding values for the free atom. In this case the solid line is the reference to indicate the values where there will not be any perturbation of the atomic splitting caused by the presence of the surface. low-spin state decreases when the support is present. This is a clear indication that even if the metal-support interaction tends to stabilize the low-spin state with respect to the isolated atom, this effect is not in general enough to quench the spin. A final point concerns the difference in the equilibrium distance perpendicular to the surface already noticed for $\mathrm{Ni}^{14}$

Except for $\mathrm{V}, \mathrm{Fe}$, and $\mathrm{Co}$, which show almost no variation, the metal atom approaches the surface when the spin is quenched. This is due to the formation of the bonding with the surface although from the results in Table III it is clear that this is in general energetically not favored. This explanation is reinforced by inspection of the atomic transition energies from the ground to the lowest excited state which is rather small for $\mathrm{V}, \mathrm{Co}$, and $\mathrm{Ni}: 0.24 \mathrm{eV}$ from $d^{3} s^{2}$ to $d^{4} s^{1}$ in $\mathrm{V}, 0.42$ for $d^{7} s^{2}$ to $d^{8} s^{1}$ in $\mathrm{Co}$, and $-0.03 \mathrm{eV}$ from $d^{8} s^{2}$ to $d^{9} s^{1}$ for $\mathrm{Ni}$, compared to values larger than 0.8 for the rest. ${ }^{32}$ Therefore, for $\mathrm{V}, \mathrm{Co}$, and $\mathrm{Ni}$ the adsorption energy is enough to overcome the energy necessary to quench the spin, whereas in the remaining atoms in the series the corresponding transition energies are considerably too large for the quenching to occur.

\section{CONCLUSIONS}

The adsorption of first-row transition-metal atoms on $\mathrm{MgO}(100)$ has been studied using periodic and cluster models. The periodic calculations are in agreement with recent experimental studies ${ }^{29}$ indicating that the trend in adsorption energies along the series is almost parallel to that corresponding to the bulk metal cohesive energy. This indicates that the changes in metal-oxide and metal-metal interactions along the first-row transition metal are governed by atomic properties. Both, periodic and cluster model calculations of the first-row transition-metal atoms adsorbed on $\mathrm{MgO}(100)$ indicate that, except for $\mathrm{Ni}$ at the GGA level, the number of unpaired electron is maintained as in the isolated metal atom. This is an important conclusion showing that at very low coverage the atomic character is preserved. Consequently, theoretical calculations should explicitly consider the openshell character of the adsorbate. This can be achieved either by using spin-polarized cluster or periodic calculations or by using spin eigenfunctions within the cluster approach.

Except for V, Co, and Ni, both PW91 and B3LYP predict the proper electronic configuration of the isolated atoms and this is maintained upon interaction with the oxide surface. PW91 fails to predict the proper ground state of $\mathrm{V}$ and $\mathrm{Ni}$, but within a given method the predicted ground states for the isolated and adsorbed atoms are the same. B3LYP properly predicts the ground states of all first-row transition atoms and, in addition, the high- to low-spin transition considered in the present work is remarkably close to experiment except for Ni which appears to be a rather special case. An important general feature is that this transition energy is always lower in the adsorbed state. However, this energy lowering does not seem to be enough to quench the magnetic moment of the adsorbed metal atom except for $\mathrm{V}, \mathrm{Co}$, and $\mathrm{Ni}$, where the first excited state lies within $\sim 0.5 \mathrm{eV}$ (Ref. 32) from the ground state. Hence, the number of unpaired electrons in the 
adatom tends to be the same as in the atomic ground state in the gas phase.

To summarize, the present study establishes the following (a) The computation of the interaction energy of transition metal atoms with regular anionic sites of $\mathrm{MgO}(100)$ requires explicitly spin polarization to be taken into account. (b) Except for $\mathrm{V}, \mathrm{Co}$, and $\mathrm{Ni}$, the metal-support interactions is not enough to quench the atomic magnetic moment and, consequently, upon adsorption all atoms maintain their atomic spin. (c) In any case, the high- to low-spin energy transition is always lower in the adsorbed state.

\section{ACKNOWLEDGMENTS}

This research was supported by the Spanish MCyT Grant No. BQU2002-04029-C02-01, by the French Ministry of

*Email address: f.illas@qf.ub.es

${ }^{1}$ Chemisorption and Reactivity on Supported Clusters and Thin Films, Vol. 331 at NATO Advanced Study Institute, Series E: Physics, edited by R. M. Lambert and G. Pacchioni (Kluwer, Dordrecht, 1997).

${ }^{2}$ G. Pacchioni, and F. Illas, in Catalysis and Electrocatalysis at Nanoparticle Surfaces, edited by E. Savinova et al., (Dekker, New York, in press).

${ }^{3}$ B. C. Gates, Catalytic Chemistry (Wiley, New York, 1992).

${ }^{4}$ C. R. Henry, Surf. Sci. Rep. 31, 231 (1998).

${ }^{5}$ M. Valden, X. Lai, and D. W. Goodman, Science 281, 1647 (1998).

${ }^{6}$ P. L. Hansen, J. B. Wagner, S. Helveg, J. R. Rostrup-Nielsen, B. S. Clausen, and H. Topsøe, Science 295, 2053 (2002).

${ }^{7}$ J. T. Ranney, D. E. Starr, J. E. Musgrove, D. J. Bald, and C. T. Campbell, Faraday Discuss. 114, 195 (1999).

${ }^{8}$ K. Højrup Hansen, T. Worren, S. Stempel, E. Lægsgaard, M. Bäumer, H.-J. Freund, F. Besenbacher, and I. Stensgaard, Phys. Rev. Lett. 83, 4120 (1999).

${ }^{9}$ B. Hammer, L. B. Hansen, and J. Nørskov, Phys. Rev. B 59, 7413 (1999).

${ }^{10}$ N. Lopez and F. Illas, J. Phys. Chem. 102, 1430 (1998).

${ }^{11}$ N. Lopez, F. Illas, N. Rösch and G. Pacchioni, J. Chem. Phys. 110, 4873 (1999).

${ }^{12}$ I. Yudanov, G. Pacchioni, K. Neyman, and N. Rösch, J. Phys. Chem. B 101, 2786 (1997).

${ }^{13}$ J. Goniakovski, Phys. Rev. B 59, 11047 (1999).

${ }^{14}$ A. Markovits, M. K. Skalli, C. Minot, G. Pacchioni, N. Lopez, F. Illas, J. Chem. Phys. 115, 8172 (2001).
Education through the GDR "Dynamique Moléculaire Quantique Appliqué a la Catalyse" and, in part by Generalitat de Catalunya Grant No. 2001SGR-00043. Part of the computer time was provided by the Center de Supercomputació de Catalunya, CESCA, and Center Europeu de Paral.lelisme de Barcelona, CEPBA, through a grant from the Universitat de Barcelona. The periodic calculations were carried on the SP3 parallel machine of the CEPBA-IBMResearch-Institute, CIRI, of Barcelona and on the CNRSIDRIS computer facilities. N. L. thanks the Spanish MCyT for supporting her work through the Ramon y Cajal program and A.M. is grateful to the European Community for financing his stay in Barcelona through IHP, program under Contract No. HPRI-CT-1999-00071 held by the CESCA/ CEBPA.
${ }^{15}$ N. Lopez, J. C. Paniagua, and F. Illas, J. Chem. Phys. 117, 9445 (2002).

${ }^{16}$ O. Robach, G. Renaud, and A. Barbier, Phys. Rev. B 60, 5858 (1999).

${ }^{17}$ J. P. Perdew and Y. Wang, Phys. Rev. B 45, 13244 (1992).

${ }^{18}$ J. P. Perdew, J. A. Chevary, S. H. Vosko, K. A. Jackson, M. R. Pederson, D. J. Singh, and C. Fiolhais, Phys. Rev. B 46, 6671 (1992).

${ }^{19}$ A. D. Becke, J. Chem. Phys. 98, 5648 (1993); C. Lee, W. Yang, and R. G. Parr, Phys. Rev. B 37, 785 (1988).

${ }^{20}$ E. G. Moroni, G. Kresse, J. Hafner, and J. Furthmüller, Phys. Rev. B 56, 15629 (1997).

${ }^{21}$ D. Vanderbilt, Phys. Rev. B 41, 7892 (1990).

${ }^{22}$ G. Kresse and J. Hafner, J. Phys.: Condens. Matter 6, 8245 (1994).

${ }^{23}$ P. J. Hay and W. R. Wadt, J. Chem. Phys. 82, 270 (1985).

${ }^{24}$ G. Kresse and J. Hafner, Phys. Rev. B 47, 558 (1993).

${ }^{25}$ G. Kresse and J. Furthmüller, Comput. Mater. Sci. 6, 15 (1996).

${ }^{26}$ G. Kresse and J. Furthmüller, Phys. Rev. B 54, 11169 (1996).

${ }^{27}$ M. J. Frisch et al., Gaussian 98, Revision A.11, Gaussian Inc.: Pittsburgh PA, (1998).

${ }^{28}$ V. L. Moruzzi, J. F. Janak, and A. R. Williams in Calculated Electronic Properties of Metals (Pergamon, New York, 1978).

${ }^{29}$ C. T. Campbell and D. E. Starr, J. Am. Chem. Soc. 124, 9212 (2002).

${ }^{30}$ K. Pierloot, E. Toscos, and B. O. Roos, Chem. Phys. Lett. 214, 583 (1993).

${ }^{31}$ E. J. Baerends, V. Branchadell, and M. Sodupe, Chem. Phys. Lett. 265, 481 (1997).

${ }^{32}$ http://physlab2.nist.gov/PhysRefData/contents.html. 